\title{
Editorial
}

\section{Die neue Allergenkennzeichnung}

Mit einer Übergangsfrist von 3 Jahren musste zum 13.12.14 die Lebensmittelinformationsverordnung (LMIV) in Bezug auf die Allergenkennzeichnung in Unternehmen, die lose Ware ausgeben, umgesetzt sein. Die LMIV verpflichtet Lebensmittelhersteller, 13 Gruppen von deklarationspflichtigen Allergenen und Sulfit sowohl auf verpackter Ware als auch bei loser Ware zu kennzeichnen.

Das Ziel der Kennzeichnung ist in Artikel 3 der LMIV definiert. Hier heißt es, dass durch die Bereitstellung von Informationen dem Endverbraucher die Möglichkeit gegeben werden soll, eine fundierte Kaufentscheidung zu treffen, um seine Gesundheit zu schützen. Das besondere Informationsbedürfnis allergischer Verbraucher im Hinblick auf eine Kaufentscheidung zur Auswahl sicherer Lebensmittel wird in den Erwägungsgründen 24 und 48 als Begründung für die Allergenkennzeichnung dem eigentlichen Gesetzestext vorangestellt.

Die bereits bestehenden Regelungen zur Allergenkennzeichnung vorverpackter Lebensmittel, dass bestimmte, festgelegte Auslöser von Allergien und Unverträglichkeiten immer gekennzeichnet werden müssen, wenn sie als Zutat verwendet werden, bleibt bestehen. Allergene werden in der LMIV als fester Bestandteil der verpflichtenden Kennzeichnungselemente (Artikel 9, Abs. 1, Buchstabe c) aufgenommen. Ebenfalls identisch bleibt die Liste der 14 zu kennzeichnenden Auslöser von Allergien und

\footnotetext{
${ }^{1}$ LMIV, Artikel 13, Absatz 1, Darstellungsform
} der verpflichtenden Angaben
Unverträglichkeiten, die in Anhang II zu finden sind.

In Ergänzung zu der seit 2005 bestehenden Allergenkennzeichnung bei vorverpackten Lebensmitteln ist nach den Anforderungen der LMIV neu, dass Allergene auf jeder für den Endverbraucher produzierten Verpackung spätestens seit dem 13.12.14 im Zutatenverzeichnis hervorgehoben werden müssen. Dies kann z.B. durch eine andere Schriftart wie z.B. Fettdruck, eine farbige Hinterlegung oder durch Unterstreichen erfolgen.

Weiterhin neu ist gemäß Artikel 44 „Einzelstaatliche Vorschriften für nicht vorverpackte Lebensmittel“, dass die Angabe von Auslösern von Allergien und Unverträglichkeiten auch bei loser Ware, also Lebensmitteln, die beispielsweise in Bäckereien, Fleischereien, Eisdielen, Frischetheken im Supermarkt, in Restaurants, Kantinen, Krankenhäusern oder im Rahmen der Kita oder Schulverpflegung abgegeben werden, verpflichtend wird.

Für verpackte und lose Ware schreibt der europäische Gesetzgeber vor, dass die Allergeninformation „an gut sichtbarer Stelle deutlich, gut lesbar und gegebenenfalls dauerhaft" angebracht werden muss. Die Allergeninformation darf dabei ,in keiner Weise durch andere Angaben oder Bildzeichen oder sonstiges eingefügtes Material verdeckt, undeutlich gemacht oder getrennt werden, und der Blick darf nicht davon abgelenkt werden." ${ }^{1}$ Generell muss die Allergeninformation unter genauer Bezugnahme zu den in Anhang II genannten Bezeichnungen der Allergene erfolgen.
Darüber hinaus ermöglicht die LMIV die Ausgestaltung der Art und Weise der Allergenkennzeichnung bei loser Ware auf nationaler Ebene. In Deutschland macht der Deutsche Allergie- und Asthmabund sich für eine schriftliche Dokumentation der Allergieauslöser stark, die dem allergischen Verbraucher zur Ansicht zur Verfügung steht.

Weiterhin nicht geregelt bleibt das mögliche unbeabsichtigte Vorkommen von Allergenen in Lebensmitteln, die sogenannte „Spurenkennzeichnung“. Jedoch ist eine mögliche zukünftige Regelung in Artikel 36 der LMIV angelegt.

\section{Fazit}

Insgesamt wird die Information für Allergiker durch die Neuerungen der LMIV sowohl bei verpackter Ware als auch durch die Erweiterung auf die nicht vorverpackten Lebensmittel deutlich verbessert. Allerdings zeigt sich in der Praxis, dass das Bewusstsein für die Allergenthematik bei Anbietern loser Ware sehr unterschiedlich und teilweise noch sehr gering ist, sodass es sicherlich noch eine Weile dauern wird, bis allergische Verbraucher tatsächlich auf eine umfassende und verlässliche Information durch die Umsetzung der neuen EUGesetzgebung zählen können.

\section{Anja Pruban, FIS Europe \\ Dr. Sylvia Pfaff, FIS Europe \\ Sabine Schnadt, Deutscher Allergie- und Asthmabund}

